\title{
Design aspects of medical laser devices
}

\author{
Jui-Teng Lin* \\ New Vision Inc, Taipei, Tawan
}

\begin{abstract}
This Editorial Review will provide and analyze certain key design aspects for effective (and marketable) medical laser devices, including: (i) the selection of laser wavelength (defining the types of therapy or diagnosis); (ii) the control penal functions (defining the choice of operations); (iii) the energy delivery means (defining the treatment target); and (iv) efficacy of the devices using a fiber-coupled multiple-wavelength system for deeper cancer therapy.
\end{abstract}

\section{Introduction}

Fundamentals and applications of medical lasers for various clinical procedures including dermatology and plastic surgery, wound healings, nerve stimulation, dentistry, cancer therapy, and ophthalmic surgeries were reviewed recently [1]. The laser spectra of UV (190400) um, visible (400-700) nm, near-IR (700-2900) nm, and mid-IR (3-5) um having various penetration depths (from determine the procedures are thermal or non-thermal or combined effect. invasive or noninvasive. Diode lasers have been widely used in many surgical procedures including soft tissue cutting, coagulation and cancer thermal therapy. Various photosensitizers are presented in matching the laser absorption wavelengths. Lasers have been used for various medical procedures including dermatology, plastic surgery, wound healings, nerve stimulation, dentistry, ophthalmology and many other therapeutic and surgical procedures [1]. Combining the nanoparticles and photosensitizers, diode lasers have been also used for cancer diagnosis and therapy [2-4]. The key design aspects for medical laser devices, including: (i) the selection of laser wavelength; (ii) the control penal functions; (iii) the energy delivery means, and (iv) the efficacy of the devices will be analyzed.

\section{The selection of laser wavelength}

Laser-tissue (or other media) interaction, in general, could be categorized into three processes: (a) pure thermal, (b) non-thermal, and (c) combined thermal and non-thermal effects. These processes are governed by not only the tissues (media) optical properties but also the laser parameters such as its wavelength, energy, intensity, pulse-width, repetition rate and the operation modes, continuous wave $(\mathrm{CW})$ or pulsed mode. Laser with very short wavelength such as $(154-193) \mathrm{nm}$, could ablate tissues without causing too much heat. Other short pulsed lasers can ablate matter via so-called plasma-assisted process. Various fiber structures for effective delivery of the laser energy to the treated areas are also critical in specific applications.

Lasers in the near infrared (IR), wavelength of $(750-1200) \mathrm{nm}$, have deeper tissue penetration depths than that of visible lasers. Therefore, near IR lasers are good candidates for procedures which need deep penetrations such as hair removal [6] and nano-gold mediated cancer therapy. On the other hand, visible lasers $(430-680) \mathrm{nm}$ with strong absorption in blood and color-dyes have been used for phototherapy of oral cancer, retina deceases and tattoo removal. Mid-IR lasers (1.9-3.0) um and (9.3 - 10.6) um with strong absorption in water and tissue have been used for super surface procedures or ablative type procedures such as soft and hard tissue ablation. Other IR lasers (1.3-1.6) um have been used for so called minimally invasive procedures such as resurfacing due to their smaller tissue absorption than that of mid-IR lasers [1].

As shown in Figure 1 (top), tissue penetration depth (d) of various lasers depends on their wavelength, or their tissue/water absorption coefficients (A), where $A$ is inverse proportional to $d$ (i.e. $d=1 / A$ ). Figure 2 (low) shows the absorption spectrum in water (or tissue) having 3 major peaks at $1.45 \mathrm{um}, 1.93 \mathrm{um}$ and $2.94 \mathrm{~nm}$, besides the high absorption of $\mathrm{CO}_{2}$ lasers (9.6 to $10.6 \mathrm{um}$.

The generalized Lambert-Beer law defines the laser intensity profile in an absorbing medium by Lin [5] as follows

$$
\begin{aligned}
& \mathrm{I}(\mathrm{z}, \mathrm{t})=\exp [-\mathrm{A}(\mathrm{z}, \mathrm{t})] \\
& \mathrm{A}(\mathrm{z}, \mathrm{t})=\int\left[a C(z, t)+b \mathrm{C}^{\prime}(z)+Q\right] d z
\end{aligned}
$$

where $C(z, t)$ is the concentration of the photosensitize; $a^{\prime}=a q$ is the coupling constant with $q$ being the quantum yield; $\mathrm{Q}$ is the tissue absorption coefficient; $a$ and $b$ are the extinction coefficient of the photosensitize and its photolysis products. Both analytic and numerical solutions of Eq. (1) have been presented by Lin et al in cancer therapy $[3,4]$ and in photo polymerizations for crosslinking process [5]. For the special that the laser is only absorbed by the medium without the photosensitize, or for constant photosensitize concentration, Eq. (1) reduces to the conventional Lambert-Beer law which is typically used in photothermal therapy (PTT). In general, $\mathrm{A}(\mathrm{z}, \mathrm{t})$ is a dynamic parameter and characterized by the chemical kinetic of the photosensitize depleted by the laser and has been used for photodynamic therapy (PDT).

\section{Laser energy delivery}

Most medical devices require a fiber delivery and/or a handheld "pen"-type piece for operational convenience. Depending on laser power and beam spot needed, commercially available fibers have typical size (core) of 200-600 um, and length of 1.0-3.0 meters. For examples,

Correspondence to: Jui-Teng Lin, New Vision Inc., 5F, No. 27, Lane 10, Jiuquan St. Da-tung Dist. Taipei, Taiwan, Tel: (407) 699-4540; E-mail: jtlin55@gmail.com Received: April 03, 2017; Accepted: April 26, 2017; Published: April 29, 2017 

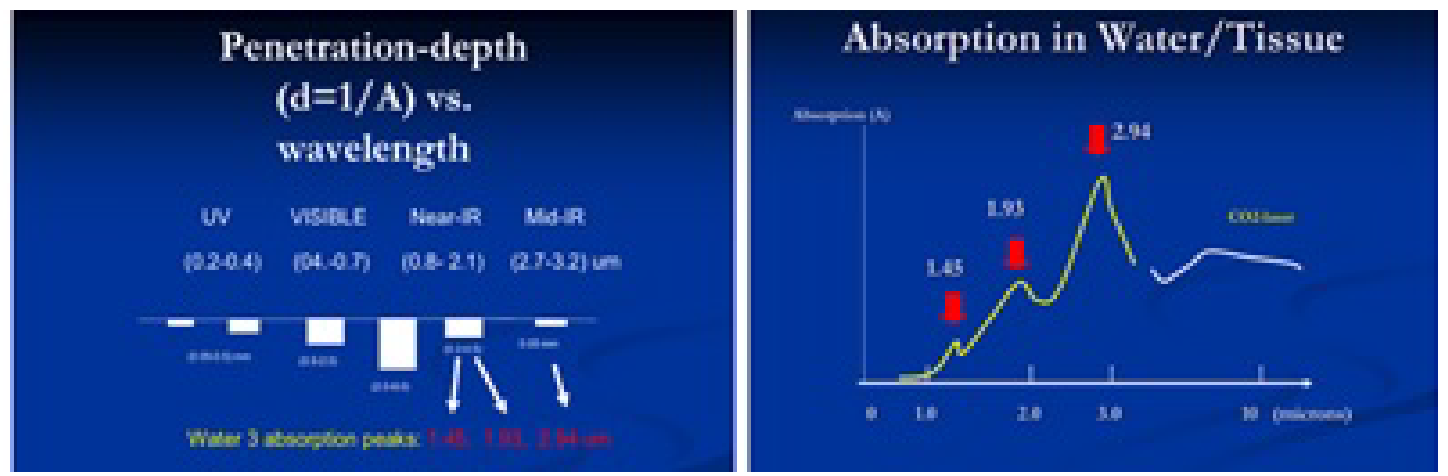

Figure 1. Tissue penetration depth (d) of lasers at various wavelength;(low) the absorption spectrum in water (or tissue).

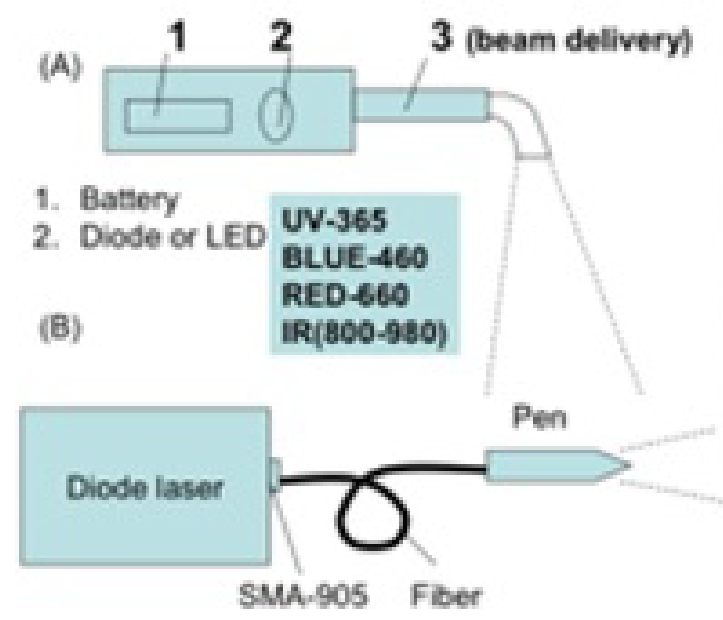

Tapered Fiber

(C)

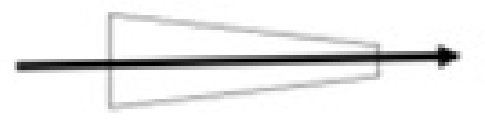

(D)

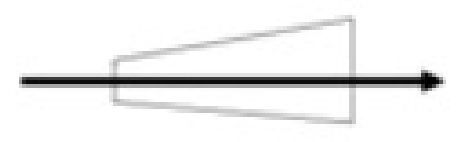

Figure 2. Various hand piece designs.

multiple uses dental fiber, 3.0 meter length is typical. Tapered fibers are customized designs for applications such as high power Er:YAG laser system and UV pulsed lasers.

As shown in Figure 2, various hand piece designs with batteryoperated LED or diode laser. Two types of tapered fiber designs: one for controller a larger beam to a smaller output end, which may be used for efficient power coupling, from larger core to smaller core, which also can avoid fiber input end face due to high-power or UV wavelength, in the case of pulsed lasers The reversed design (D) is for larger targeting area and for smaller beam divergence (to achieve deeper penetration of the light (LED or lasers) into the targeted tissues.

Figure 2 shows the detail structures of the "laser pen" connected to the fibers. This hand piece consisting (se A \&B) of one or a set of focusing lenses will be designed in order to adjust the laser spot size on the target area, either collimated or focused. A stopper is a common practice for controlling the action area at a predefined distance. Detachable, one time use fiber tip (see C) and angled fiber (see D) are also useful in many clinical uses, such as for dental and eye surgeries.

\section{Multiple wavelength system}

As show in Figure 3, there-wavelength diode laser system was designed for cancer therapy (with nanogold in rod shape as the absorber), where near IR diode lasers at $808 \mathrm{~nm}, 905 \mathrm{~nm}$ and $980 \mathrm{~nm}$ are coupled to a single fiber. Selection the output of one or all lasers can be controlled when needed. As show by Eq. (1) and Figure 3, the absorption depth is inverse proportional to the absorption coefficients at a specific wavelength (w1, w2, w3). Therefore, deeper and 3 -dimemnsional cancer tumor can be treated using various wavelength (w1<w2<w3), where $\mathrm{w} 1=808 \mathrm{~nm}$ (having the shallow absorption) matching the peak absorption of the gold nanorod [3], whereas the other off-peak wavelength (w2 and W3) have deeper penetration due to smaller absorption coefficients. Above described design offers faster, deeper laser therapy particularly for large volume tumor.

Typical medical laser device requires clinically useful functions including: (a) adjustable laser power, (b) selection of $\mathrm{cw}$ or pulsed modes, (c) pulse-width and repetition rate and (e) a footswitch for surgeons operation. Additionally, to meet the CE-mark or FDA approval, an emergent switch is also required.

Diode lasers for various surgical procedures including soft tissue cutting, coagulation and cancer thermal therapy using various photosensitizers matching the laser absorption wavelengths [1]. The principles and applications of photothermal therapy (PTT) and photodynamic therapy (PDT) were discussed previously [3].

Various photosensitizers are available for the absorption of lasers in visible $(630-700) \mathrm{nm}$, and near-IR (700-1000) $\mathrm{nm}$. These lasers are commercially available; dye lasers (at about $665 \mathrm{~nm}$ ) pumped by a green laser; Nd:YAG (at $1064 \mathrm{~nm}$ ); diode lasers (630-1100) nm; tunable Ti:sapphire laser (690-1100) nm; Alexandrite laser (720-800) $\mathrm{nm}$. Tunable near-IR source may be also generated from an optical parametric oscillation (OPO) or amplification (OPA), where a green laser (at about $532 \mathrm{~nm}$ ) may be used as a pump to produce tunable (900-1300) nm near-IR output. In addition, high-brightness LED in visible (550-680) $\mathrm{nm}$ are also available for PDT using 5-ALA as the photosensitizer [3].

The design of multiple-wavelengths laser system shall partially overcome the issues of gold nanorod's non-uniform and multiple thermal medium for a 3-dimensional-therapy, in which various absorption penetration depths are available via the fiber-coupled multiple-wavelength laser simultaneously targeting the cancer tumors. 
As shown by Figure 5, combining PDT and PTT using nanogold and various photosensitizers for cancer therapy was discussed previosuly [3]. The critical factors of the synergistic therapy efficiency including: the concentration of the initiator (nanogold or photosensitizers) in the treated medium, the wavelength, energy and the irradiation period of the light applied to the medium.

\section{Conclusion}

Various key design aspects of medical laser devices, including the selection of laser wavelength and the energy delivery means and panel functions are discussed. Maximal cancer phototherapy (either PTT or PDT) efficacy may be achieved by using a fiber-coupled multiplewavelength system for deeper cancer therapy.

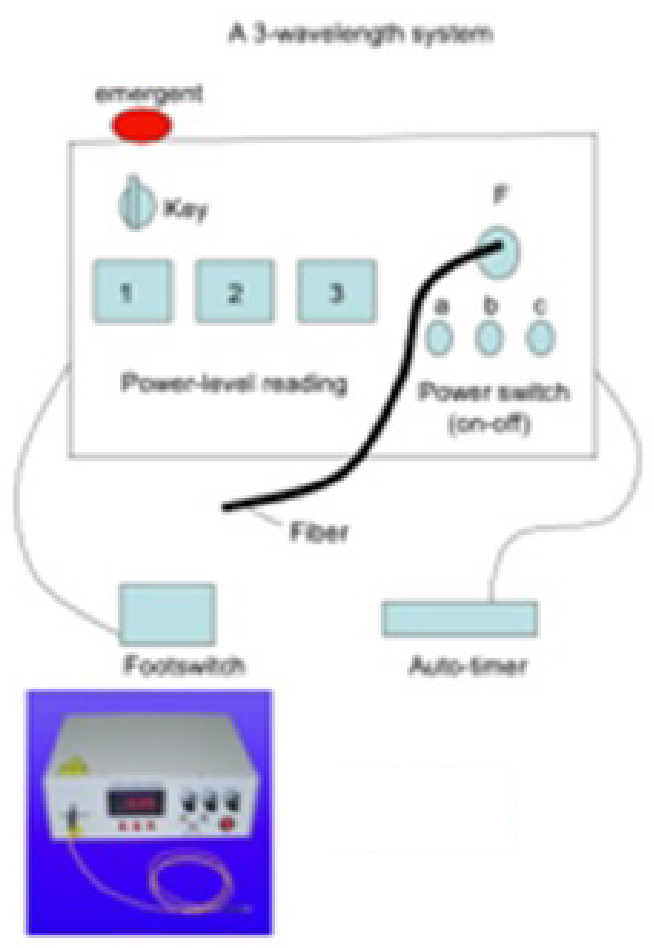

Figure 3. Three-wavelength diode laser system for cancer therapy (with nanogold as the absorber), where near IR diode lasers at $808 \mathrm{~nm}, 905 \mathrm{~nm}$ and $980 \mathrm{~nm}$ are coupled to a single fiber.

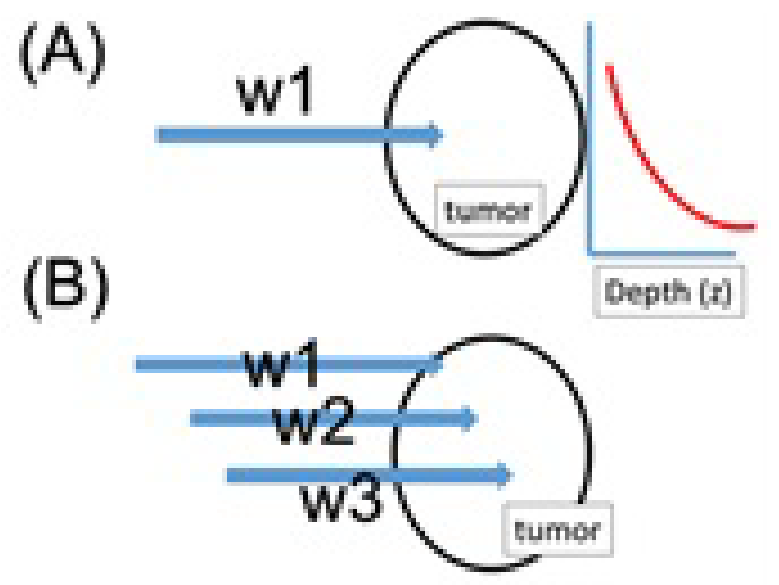

Figure 4. Cancer phototherapy system with (A) single wavelength, and (B) 3 wavelengths Also shown is the exponential decaying of the laser intensity inside the treated tumor.
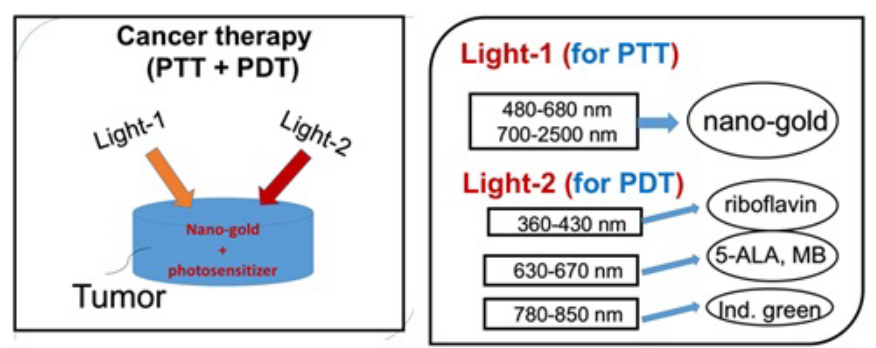

Figure 5. Combining PDT and PTT uisng nanogold and various photosensitizers [3].

\section{References}

1. Lin JT (2016) Progress of medical lasers: fundamentals and applications. Med Devices Diagn Eng 1: 36-41.

2. Lin JT (2012) Chiang S, Lin GH et al. In vitro photothermal destruction of cancer cells using gold nanorods and pulsed-train near-infrared lase. J Nanomaterials.

3. Lin JT (2017) Analysis of the efficiency of photothermal and photodynamic cancer therapy via nanogolds and photosensitizers. J Cancer Research Update pp: 12-18.

4. Huang X, El-Sayed MA (2010) Gold nanoparticles: Optical properties and implementations in cancer diagnosis and photothermal therapy. $J$ Advanced Research 1: $13-28$

5. Lin JT (2016) Combined analysis of safety and optimal efficacy in UV-light-activated corneal collagen crosslinking. Ophthalmology Research 6: 1-14.

Copyright: $(2017$ Lin JT. This is an open-access article distributed under the terms of the Creative Commons Attribution License, which permits unrestricted use, distribution, and reproduction in any medium, provided the original author and source are credited. 